\title{
Effects of melatonin on liver function and lipid peroxidation in a rat model of hepatic ischemia/reperfusion injury
}

\author{
WEN-SHENG DENG, QING XU, YE LIU, CHUN-HUI JIANG, HONG ZHOU and LEI GU \\ Department of Gastrointestinal Surgery, Renji Hospital, School of Medicine, \\ Shanghai Jiaotong University, Shanghai 200127, P.R. China
}

Received March 12, 2014; Accepted October 9, 2015

DOI: $10.3892 /$ etm.2016.3160

\begin{abstract}
The present study aimed to investigate the effects of melatonin (MT) on liver function and lipid peroxidation following hepatic ischemia-reperfusion injury (IRI). A total of 66 male Sprague-Dawley rats were randomly assigned into three groups: Normal control (N) group, ischemia-reperfusion (IR) group and the MT-treated group. A hepatic IRI model was developed by blocking the first porta hepatis, and subsequently restoring hepatic blood inflow after $35 \mathrm{~min}$. Following reperfusion, changes in the levels of alanine aminotransferase (ALT), aspartate aminotransferase (AST), lactate dehydrogenase (LDH), malondialdehyde (MDA), superoxide dismutase (SOD) and glutathione (GSH) were detected by a chemical method at various time points. In the MT group, the MDA levels were significantly reduced $(\mathrm{P}<0.05)$ at all time points, as compared with the IR group. Furthermore, SOD activity was significantly increased $(\mathrm{P}<0.05)$ in the MT group, as compared with the IR group at all time points; and the levels of GSH in the MT group were significantly higher $(\mathrm{P}<0.05)$ than those of the IR group at 2, 4, and $8 \mathrm{~h}$ post-reperfusion. The levels of ALT, AST and LDH were significantly reduced in the MT group at each time point, as compared with that of the IR group $(\mathrm{P}<0.05)$. In conclusion, MT exhibits potent antioxidant properties that may create favorable conditions for the recovery of liver function following IRI.
\end{abstract}

\section{Introduction}

In order to effectively control bleeding during hepatobiliary surgery, hepatic portal occlusion is often used, as it can temporarily interrupt the portal vein and hepatic artery inflow. This method is widely accepted since it is clinically simple, practical and effective; however, there is an inherent risk of

Correspondence to: Dr Lei Gu, Department of Gastrointestinal Surgery, Renji Hospital, School of Medicine, Shanghai Jiaotong University, 160 Pujian Road, Shanghai 200127, P.R. China

E-mail: renjigulei@163.com

Key words: melatonin, liver ischemia-reperfusion injury, liver function, lipid peroxidation subsequent liver ischemia-reperfusion injury (IRI). Therefore, in recent years, clinical research in the field of hepatic surgery has focused on how to reduce blood loss and improve the safety of hepatic portal occlusion, whilst simultaneously reducing the risk of liver IRI.

Previous studies investigating tissues and organs such as the brain, heart, kidney and retina have demonstrated that numerous free radicals are produced during IRI, when the endogenous radical scavengers cannot function appropriately, leading to cell damage $(1,2)$. Therefore, investigating effective drugs that alleviate IRI-induced functional liver damage, and improve the postoperative survival rate, has become a research hotspot in the field of hepatic surgery. Melatonin (MT) is a neural endocrine hormone that is secreted by the pineal gland (PG), which exerts known antioxidant, antitoxic, anti-stress and anti-inflammatory effects $(3,4)$. Previous studies have demonstrated that MT has a protective role in IRI of the brain, heart, kidney and retina (5); however, its protective effects in liver IRI and the underlying mechanism require further research. An animal model of hepatic portal occlusion was used in the present study. MT was administered in advance, and the influence and significance of MT on antioxidant capacity, lipid peroxidation damage and liver function in liver IRI was investigated.

\section{Materials and methods}

Experimental animals. A total of 66 male Sprague-Dawley rats were used in the present study, and were purchased from the Laboratory Animal Department of Shanghai Jiaotong University School of Medicine (Shanghai, China). The rats were aged 3-6 months, and weighed $220 \pm 30 \mathrm{~g}$. The experimental procedures of the present study conformed to the guidelines outlined in the guide for the Care and Use of Laboratory Animals and were approved by the Research Ethics Committee of Renji Hospital [Shanghai, China; approval no. SYXY(hu)2011-0121]. The rats were randomly assigned into three groups: i) The normal control group ( $\mathrm{N}$ group; $\mathrm{n}=6$ ), ii) the ischemia-reperfusion (IR) group $(n=30)$; iii) and the MT-treated group $(n=30)$. The IR and MT groups were divided into five subgroups $(n=6)$, according to the following time points: $35 \mathrm{~min}$ of ischemia, and 2, 4, 8 or $24 \mathrm{~h}$ of reperfusion.

Animal model and sample collection. The rats were fasted overnight prior to surgery, and were subsequently anesthetized with 
$3 \%$ pentobarbital sodium $(1 \mathrm{ml} / \mathrm{kg}$; Sigma-Aldrich, St. Louis, MO, USA) via intraperitoneal injection. Following this, the rats were fixed in the supine position on a regularly disinfected and surgically draped operating table. A straight $\sim 2 \mathrm{~cm}$ incision was made in the middle of the upper abdomen, along which the abdomen was cut step-by-step. The peri-hepatic ligament was carefully dissected to expose the hepatoduodenal ligament. In order to completely block the portal vein, hepatic artery and bile duct, an atraumatic hemostatic clamp was used to block the first porta hepatis, according to the Pringle manoeuver (6). The clamp was released after $35 \mathrm{~min}$ to recover the inflow of hepatic blood, and the abdomen was subsequently sutured. In the MT group, intraperitoneal injections of $10 \mathrm{mg} / \mathrm{kg}$ MT [1 $\mathrm{ml}$ (ip)] were administered 35 and 70 min prior to ischemia, as well as at the early stage of reperfusion, and at 1 and $2 \mathrm{~h}$ post-reperfusion (7-12).

Portal vein blood samples $(1 \mathrm{ml})$ were obtained from the rats in each group at various time points $(n=6$ at each time point). The time points were as follows: Prior to ischemia, $35 \mathrm{~min}$ of ischemia, and 2, 4, 8 and $24 \mathrm{~h}$ post-reperfusion. The rats were sacrificed immediately after each time point. Blood samples were placed in a sterile Eppendorf tube without pyrogen for $20 \mathrm{~min}$ prior to centrifugation at 4,000 $\mathrm{x} \mathrm{g}$ for $10 \mathrm{~min}$. The serum was subsequently separated and stored at $-30^{\circ} \mathrm{C}$. A total of $1 \mathrm{~g}$ incised liver tissue was rinsed with normal saline (NS) at $0^{\circ} \mathrm{C}$, and stored in a liquid nitrogen tank for inspection; whereas another $1 \mathrm{~g}$ of incised liver tissue was fixed with $10 \%$ formaldehyde solution for inspection.

Instruments. The experimental table, atraumatic vascular clamp, scissors, forceps, and hemostatic forceps were purchased from Shanghai Medical Equipment Works Co. (Shanghai, China). A high speed centrifuge and an ultraviolet (UV) spectrophotometer were purchased from Thermo Fisher Scientific, Inc. (Waltham, MA, USA); an enzyme-linked immunosorbent assay plate reader was obtained from Bio-Rad Laboratories, Inc. (Hercules, CA, USA); and a CL-7150 automatic biochemical analyzer (ACA) was purchased from Shimadzu Corporation (Kyoto, Japan).

Reagents. Malondialdehyde (MDA), superoxide dismutase (SOD) and glutathione (GSH) peroxidase assay kits and the reagents for total protein quantification, including biuret, were purchased from Nanjing Jiancheng Bioengineering Institute (Nanjing, China). Reagent configurations were prepared according to the manufacturer's protocol. MT was purchased from Sigma-Aldrich.

Determination of liver function. Alanine aminotransferase (ALT), aspartate aminotransferase (AST) and lactate dehydrogenase (LDH) levels were measured using a CL-7150 ACA according to the manufacturer's protocol. These experiments were performed as previously described (13).

Determination of MDA using thiobabituric acid. Thiobabituric acid was used to detect MDA levels as the two compounds form a red product through condensation, with a maximum absorption peak of $532 \mathrm{~nm}$. The tissue homogenate was prepared as follows: $100 \mathrm{mg}$ liver tissue was harvested and supplemented with $9 \mathrm{X}$ physiological saline prior to ultrasonic homogenization for $20 \mathrm{sec}$ and subsequent centrifugation at $300 \mathrm{x} \mathrm{g}$ at $4^{\circ} \mathrm{C}$ for
$15 \mathrm{~min}$. The resultant supernatant was tested using a standard, and reagents 1-3. According to the manufacturer's protocol, the standard, anhydrous ethanol and sample $(0.1 \mathrm{ml}$ each) were added into the standard tube, test tube and test control tube, respectively; and $0.1 \mathrm{ml}$ reagent 1 was subsequently added into each tube. Following this, the tube rack was agitated in order to mix the reagents, and $3 \mathrm{ml}$ reagent 2 and $1 \mathrm{ml}$ reagent 3 were added to the standard control and test tubes, respectively; whereas $3 \mathrm{ml}$ reagent 2 and $50 \%$ glacial acetic acid $(1 \mathrm{ml})$ were added into the test control tube, and mixed in a swirl mixer, with the tube mouth tightly covered with plastic wrap with a small hole. The optical density (OD) values of each tube were measured at $532 \mathrm{~nm}$ using a UV spectrophotometer. The MDA content was subsequently calculated according to the formula outlined in the manufacturer's protocols.

Determination of SODlevels, using the xanthine oxidase method. Utilizing the specific inhibition of SOD on superoxide anion free radicals, the xanthine oxidase method was used to determine the levels of SOD. The tissue homogenate was prepared as follows: $100 \mathrm{mg}$ liver tissue was harvested and supplemented with $9 \mathrm{X}$ physiological saline prior to ultrasonic homogenization for $20 \mathrm{sec}$ and subsequent centrifugation at $300 \mathrm{x} \mathrm{g}$ at $4^{\circ} \mathrm{C}$ for $15 \mathrm{~min}$. The resultant supernatant was tested according to the manufacturer's protocols. The following was added to the test tube: $1 \mathrm{ml}$ reagent $1,0.03 \mathrm{ml}$ sample, $0.5 \mathrm{ml}$ distilled water, $0.1 \mathrm{ml}$ reagent $2,0.1 \mathrm{ml}$ reagent 3 and $0.1 \mathrm{ml}$ reagent 4 ; whereas in the control tube, the sample was substituted with $0.03 \mathrm{ml}$ distilled water. The solutions were subsequently mixed in a swirl mixer, and incubated in a thermostatic water bath at $37^{\circ} \mathrm{C}$ for $40 \mathrm{~min}$. Once the tubes had cooled, $2 \mathrm{ml}$ chromogenic agent was added to each tube, and the solution was agitated for $10 \mathrm{~min}$. The OD values were subsequently measured at $550 \mathrm{~nm}$ using a UV spectrophotometer. SOD activity was calculated according to the formula outlined in the manufacturer's protocol.

Determination of GSH by colorimetry. GSH was detected as the yellow product formed in the reaction between sulfhydryl compounds and dithiobisnitrobenzoicacid. Briefly, the tissue homogenate was prepared as follows: $100 \mathrm{mg}$ liver tissue was harvested and supplemented with 9X physiological saline prior to ultrasonic homogenization for $20 \mathrm{sec}$ and subsequent centrifugation at $300 \mathrm{x} \mathrm{g}$ at $4^{\circ} \mathrm{C}$ for $15 \mathrm{~min}$. The resultant supernatant was tested according to the manufacturer's protocol. A total of $0.03 \mathrm{ml}$ standard, $0.03 \mathrm{ml}$ distilled water, $0.03 \mathrm{ml}$ sample and $0.03 \mathrm{ml}$ sample were added to the standard, control, test and test control tubes, respectively; and each tube was supplemented with $0.03 \mathrm{ml}$ reagent $1,1 \mathrm{ml}$ reagent 2 and $0.5 \mathrm{ml}$ reagent 3 (excluding the test control tube). Following this, the solution was agitated and incubated at room temperature for $10 \mathrm{~min}$, prior to the determination of the OD values at $412 \mathrm{~nm}$ using a UV spectrophotometer. GSH activity was calculated according to the formula outlined in the manufacturer's protocol.

Determination of protein content via the biuret method. The tissue homogenate was prepared as follows: $100 \mathrm{mg}$ liver tissue was harvested and supplemented with 9X physiological saline prior to ultrasonic homogenization for $20 \mathrm{sec}$ and subsequent centrifugation at $300 \mathrm{x} \mathrm{g}$ at $4^{\circ} \mathrm{C}$ for $15 \mathrm{~min}$. The resultant supernatant was tested using a stock solution of Coomassie 
Table I. Malondialdehyde activity in rats with ischemia-reperfusion injury (nmol/mg prot).

Time after reperfusion (hours)

\begin{tabular}{lccccc}
\cline { 2 - 5 } Group & 0 & 2 & 4 & 8 & 24 \\
\hline N & $28.02 \pm 0.88$ & - & - & - & - \\
IR & $43.40 \pm 0.97^{\mathrm{a}}$ & $58.07 \pm 0.95^{\mathrm{a}}$ & $53.38 \pm 0.67^{\mathrm{a}}$ & $45.17 \pm 0.4^{\mathrm{a}}$ & $44.73 \pm 0.75^{\mathrm{a}}$ \\
MT & $41.47 \pm 0.84^{\mathrm{a}, \mathrm{b}}$ & $56.65 \pm 0.13^{\mathrm{a}, \mathrm{b}}$ & $46.73 \pm 1.10^{\mathrm{a}, \mathrm{b}}$ & $43.08 \pm 0.48^{\mathrm{a}, \mathrm{b}}$ & $43.08 \pm 0.48^{\mathrm{a}, \mathrm{b}}$
\end{tabular}

${ }^{\mathrm{a}} \mathrm{P}<0.05$ vs. the $\mathrm{N}$ group; ${ }^{\mathrm{b}} \mathrm{P}<0.05$ vs. the IR group. $\mathrm{N}$, normal; IR, ischemia-reperfusion; MT, melatonin-treated.

Table II. Superoxide dismutase activity in rats with ischemia-reperfusion injury (U/mg prot).

Time after reperfusion (hours)

\begin{tabular}{lccccc}
\cline { 2 - 5 } Group & 0 & 2 & 4 & 8 & 24 \\
\hline $\mathrm{N}$ & $91.33 \pm 0.60$ & - & - & - & - \\
$\mathrm{IR}$ & $35.98 \pm 1.18^{\mathrm{a}}$ & $47.17 \pm 1.78^{\mathrm{a}}$ & $50.50 \pm 1.81^{\mathrm{a}}$ & $60.10 \pm 0.70^{\mathrm{a}}$ & $84.97 \pm 0.61^{\mathrm{a}}$ \\
$\mathrm{MT}$ & $41.63 \pm 0.85^{\mathrm{a}, \mathrm{b}}$ & $53.35 \pm 3.98^{\mathrm{a}, \mathrm{b}}$ & $52.94 \pm 1.72^{\mathrm{a}, \mathrm{b}}$ & $63.62 \pm 1.97^{\mathrm{a}, \mathrm{b}}$ & $86.45 \pm 0.65^{\mathrm{a}, \mathrm{b}}$
\end{tabular}

${ }^{\mathrm{a}} \mathrm{P}<0.05$ vs. the $\mathrm{N}$ group; ${ }^{\mathrm{b}} \mathrm{P}<0.05$ vs. the IR group. $\mathrm{N}$, normal; IR, ischemia-reperfusion; MT, melatonin-treated.

Brilliant Blue (CBBG250; Sigma-Aldrich), prepared for standard protein solution. A total of $0.05 \mathrm{ml}$ distilled water $+3 \mathrm{ml}$

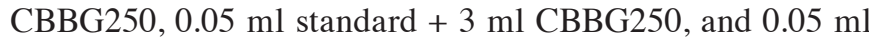
sample $+3 \mathrm{ml} \mathrm{CBBG} 250$ were added into the control, standard tube and test tubes, respectively. The solution was agitated and incubated at room temperature for $10 \mathrm{~min}$, and the OD values were measured at $495 \mathrm{~nm}$ using a UV spectrophotometer in order to calculate the protein content, based on the following formula: Protein content $=(O D$ value of test tube $/ O D$ value of standard tube) $\mathrm{x}$ standard protein content.

Statistical analysis. SPSS 17.0 software (SPSS, Inc., Chicago, IL, USA) was used to determine statistical differences. Quantitative data are expressed as the mean \pm standard deviation. One-way analysis of variance and a Student Newman-Keuls post-hoc test were performed to analyze variance between the groups. $\mathrm{P}<0.05$ was considered to indicate a statistically significant difference.

\section{Results}

Ischemia significantly increases MDA levels. At each time point, the MDA levels in the IR and MT groups were significantly increased following ischemia $(\mathrm{P}<0.05)$, as compared with pre-ischemic levels. MDA levels in the two groups peaked $2 \mathrm{~h}$ after reperfusion, and subsequently declined. At all time points, the levels of MDA were significantly decreased $(\mathrm{P}<0.05$; Table $\mathrm{I})$ in the MT group following liver ischemia, as compared with those of the IR group.

MT administration increases SOD activity following ischemia. SOD values were lower at each time point after ischemia in the IR and MT groups, as compared with the $\mathrm{N}$ group $(\mathrm{P}<0.05)$. SOD levels of the two groups were similar to those of Group $\mathrm{N}$ at $24 \mathrm{~h}$ post-reperfusion. Following liver ischemia, the SOD activities in the MT group were significantly increased $(\mathrm{P}<0.05$; Table II) at all time points, as compared with those in the IR group.

MT administration increases GSH levels following ischemia. At each post-ischemic time point, the GSH values of the IR and MT groups were significantly reduced $(\mathrm{P}<0.05)$, as compared with those of group $\mathrm{N}$; however, at $8 \mathrm{~h}$ post-reperfusion, the GSH levels of the two groups were similar to that of group $\mathrm{N}$. Furthermore, the GSH levels in group MT were significantly higher than those of the IR group at 2,4 , and $8 \mathrm{~h}$ post-reperfusion $(\mathrm{P}<0.05$; Table III $)$.

MT administration significantly decreases ALT levels following ischemia. The ALT values corresponding to $35 \mathrm{~min}$ of hepatic portal occlusion, and 2, 4, 8 and 24 h post-reperfusion, were $150.17 \pm 13.85,543.93 \pm 50.50,976.50 \pm 32.92,884.75 \pm 21.78$ and $281.87 \pm 10.75 \mathrm{IU} / 1$, respectively, in the IR group; as compared with $127.63 \pm 19.46,367.28 \pm 12.53,683.35 \pm 20.21,586.77 \pm 29.57$ and $188.08 \pm 11.09 \mathrm{IU} / \mathrm{l}$ in the MT treatment group. All of the ALT values were significantly higher than those determined in the $\mathrm{N}$ group $(46.22 \pm 8.28, \mathrm{P}<0.05)$. Furthermore, in the MT group, the ALT levels were significantly lower $(\mathrm{P}<0.05)$ than those determined in the IR group, at each time point (Fig. 1A).

MT administration significantly decreases AST levels following ischemia. The AST values corresponding to $35 \mathrm{~min}$ of hepatic portal occlusion, and 2, 4, 8 and 24 h post-reperfusion, were $974.70 \pm 53.65,1453.73 \pm 56.70,1866.53 \pm 156.46,1588.56 \pm 141.89$ and $428.76 \pm 35.23 \mathrm{IU} / 1$, respectively, in the IR group; as compared with $521.83 \pm 29.46,961.24 \pm 62.45,1345.98 \pm 167.78$, $1134.45 \pm 129.68$ and $283.79 \pm 21.43$ IU/1 in the MT group. All of these AST values were significantly higher than those determined 
Table III. Glutathione activity changes of rats with ischemia-reperfusion injury (U/mgprot).

Time after reperfusion (hours)

\begin{tabular}{lccccc}
\cline { 2 - 6 } Group & 0 & 2 & 4 & 8 & 24 \\
\hline N & $97.17 \pm 0.73$ & - & - & - & - \\
IR & $77.90 \pm 1.85^{\mathrm{a}}$ & $75.93 \pm 1.50^{\mathrm{a}}$ & $76.50 \pm 2.02^{\mathrm{a}}$ & $84.75 \pm 0.87^{\mathrm{a}}$ & $83.07 \pm 0.25^{\mathrm{a}}$ \\
MT & $76.63 \pm 1.46^{\mathrm{a}, \mathrm{b}}$ & $80.28 \pm 2.59^{\mathrm{a}, \mathrm{b}}$ & $83.35 \pm 0.29^{\mathrm{a}, \mathrm{b}}$ & $86.77 \pm 0.59^{\mathrm{a}, \mathrm{b}}$ & $82.98 \pm 1.00^{\mathrm{a}, \mathrm{b}}$ \\
\hline
\end{tabular}

${ }^{\text {a }} \mathrm{P}<0.05$ vs. the $\mathrm{N}$ group; ${ }^{\mathrm{b}} \mathrm{P}<0.05$ vs. the IR group. $\mathrm{N}$, normal; IR, ischemia-reperfusion; MT, melatonin-treated

A

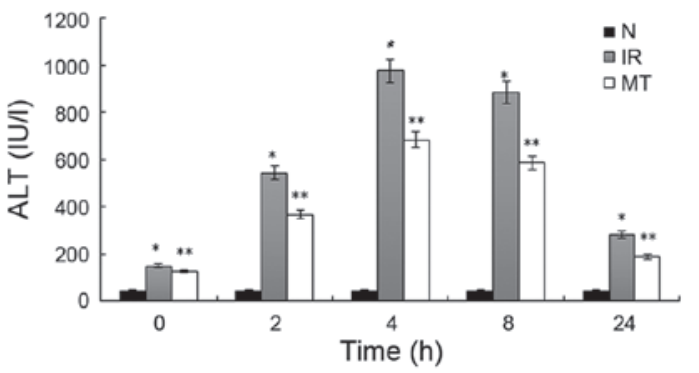

B

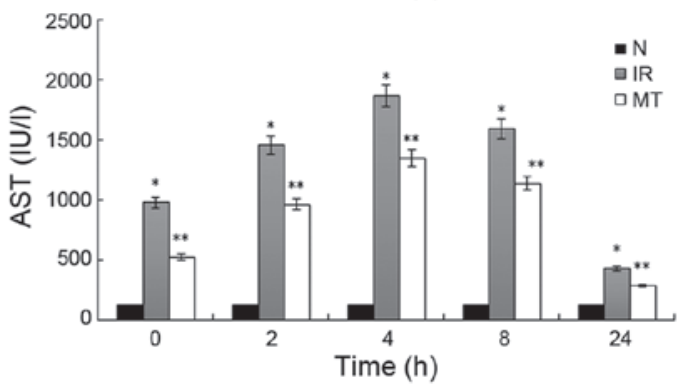

C

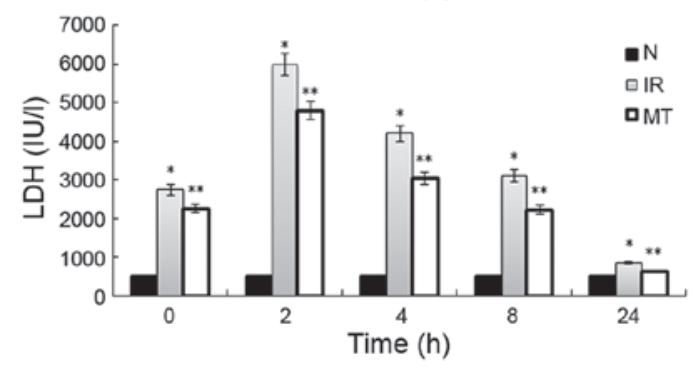

Figure 1. (A) Alanine aminotransferase, (B) aspartate aminotransferase and (C) lactate dehydrogenase levels in rats with ischemia-reperfusion injury at the indicated time points. ${ }^{*} \mathrm{P}<0.05$ vs. the $\mathrm{N}$ group; ${ }^{* *} \mathrm{P}<0.05$ vs. the IR group. $\mathrm{N}$, normal; IR, ischemia-reperfused; MT, melatonin-treated.

in the $\mathrm{N}$ group $(115.22 \pm 15.08 ; \mathrm{P}<0.05)$. Furthermore, in the MT group, the AST levels were significantly lower $(\mathrm{P}<0.05)$ than those of the IR group, at each time point (Fig. 1B).

MT administration significantly decreases LDH levels following ischemia. The LDH values corresponding to $35 \mathrm{~min}$ of hepatic portal occlusion, and 2, 4, 8 and 24 h post-reperfusion were $2749.67 \pm 278.23,5987.32 \pm 956.77,4207.65 \pm 715.54$, $3098.11 \pm 556.90$ and $856.11 \pm 130.42 \mathrm{IU} / 1$, respectively, in the IR group, as compared with $2251.23 \pm 329.61,4789.12 \pm 1053.12$, $3045.45 \pm 677.21,2229.05 \pm 629.53$ and $637.31 \pm 91.02$ in the MT group. All of the LDH values were significantly higher than those determined in the $\mathrm{N}$ group (509.56 \pm 100.67 ;
$\mathrm{P}<0.05)$. Furthermore, in the MT group, the LDH activities were significantly lower $(\mathrm{P}<0.05)$ than that of the IR group, at each time point (Fig. 1C).

\section{Discussion}

Hepatectomy is the surgical resection of the liver and is considered the main therapeutic strategy for the treatment of numerous liver diseases. Since the liver has an abundant blood supply, hepatic portal occlusion is often adopted in order to reduce hemorrhage during the operation; however, this method may lead to hepatic IRI (14).

In the present study, in order to generate a rat model of hepatic acute IRI, an atraumatic vascular clamp was used to completely block the first porta hepatis, which was reopened following $35 \mathrm{~min}$ in order to recover the hepatic blood inflow. The present study demonstrated that, at the following time points: 35 min of hepatic portal occlusion, and 2, 4, 8 and $24 \mathrm{~h}$ post-reperfusion, MDA levels were significantly increased $(\mathrm{P}<0.05)$; whereas the levels of SOD and GSH were significantly decreased $(\mathrm{P}<0.05)$ in the liver tissue, as compared with the normal group. SOD and GSH are endogenous antioxidant enzymes which clear oxygen free radicals and protect cells from oxidative damage (15). As a product of lipid peroxidation, MDA reflects the state of oxidative damage. Large quantities of oxygen free radicals may cause serious damage to the body, resulting in: DNA degradation, protein oxidation, lipid peroxidation and cell death. In the present study, SOD and GSH activities were decreased in response to IR, whereas MDA content increased, which indirectly demonstrated that oxygen free radicals were predominantly produced in this process, the endogenous antioxidant was consumed, and cells were exposed to lipid peroxidation damage.

Hepatic portal occlusion can cause hepatic ischemia and hypoxia, therefore it may affect the synthesis of mitochondrial energy and electron transport in the respiratory chain $(16,17)$, producing numerous oxygen free radicals, which cause cell damage via lipid peroxidation of biological membranes (18). Lipid peroxidation itself produces toxic substances, including MDA. Furthermore, in addition to directly damaging the structure of cells (19), hypoxia can induce disturbances in hepatic microcirculation, leading to further ischemia and anoxic liver damage (20). MT consists of both hydrophilic and hydrophobic moieties, and is synthesized by the PG, which exists in all vertebrates. In addition to regulating biological rhythms, anti-inflammatory actions and sleep onset latency, previous studies have demonstrated MT is a powerful free 
radical scavenger. MT is capable of removing various oxygen free radicals, particularly hydroxyl free radicals $(21,22)$. Its ability to clear hydroxyl radicals is 500 times stronger than benzoate, a conventional hydroxyl radical scavenger (23). A previous study (24) demonstrated that MT is able to inhibit the production of excessive free radicals in myocardial IR, and can directly eliminate the formed free radicals and the activities of its related reactants, demonstrating that MT has a protective effect in the myocardium following IR.

Under normal physiological conditions, only a small amount of oxygen free radicals are produced during normal metabolic processes. In this event, the radical is immediately cleared by the in vivo defense mechanisms in order to minimize further damage. A large quantity of oxygen free radicals are generated in the liver during IRI and, due to ischemia, the intrahepatic defense mechanisms are disordered, therefore the radical cannot be cleared effectively, thus leading to the characteristic cellular damage. The damage induced by oxygen free radicals can be summarized as follows: Peroxidation of polyunsaturated fatty acids (PUFA) in cell membranes induces crosslinking in biological macromolecules such as DNA, RNA and proteins, in addition to an oxidation reaction, which prompts the polymerization and depolymerization of polysaccharide molecules $(25,26)$. Therefore, ischemic injury is characterized by a reduction in selective PUFA and is marked by lipid peroxidation of the biological membrane, with MDA as the metabolic product of membrane lipid peroxidation. By inducing a cross-linking reaction between proteins and their respective primary amino groups, MDA leads to the destruction of cell function. Therefore, by measuring the serum content of MDA, the degree of cell membrane damage can be indirectly determined (27). In the present study, the MDA increase was significantly lower in the MT group, as compared with that of the IRI group, thus suggesting that MT may promote the degradation of oxygen free radicals into non-toxic or low toxic substances, and thus enhance antioxidant capacity.

SOD is an enzyme that contains metal cofactors, which are capable of catalyzing the dismutation of superoxide anions, thus removing oxygen free radicals and protecting cells (18). The results of the present study demonstrated that, at each time point of rat liver IRI, SOD activity in the IR group was significantly decreased, as compared with the MT group; whereas the MDA content was significantly increased, as compared with the MT group. These results demonstrated that the administration of MT may partially reverse the decline of SOD activity following ischemia, whilst simultaneously maintaining the scavenging ability of ischemic tissue to oxygen free radicals, thus reducing damage associated with lipid peroxidation. Parlaktas et al (28) and Kerem et al (29) have also shown that MT can promote the activity of SOD.

In the present study, in the early stages of IR, SOD levels were significantly reduced in the two groups, as compared with the normal group; and SOD rose gradually as time progressed. The SOD levels were increasing continuously from $8 \mathrm{~h}$ post-reperfusion, while MDA dropped to the relatively low degree from the highest point following reperfusion. The changes in SOD and MDA reflect the respective protective and destructive effects induced by IRI. SOD activity was increased in the groups without MT following reperfusion in a time-dependent manner; this may be a compensatory response to the huge increase in oxygen free radicals and MDA during reperfusion. In addition, SOD activity was increased in the MT group, as compared with the IR group, and the serum content of MDA was significantly decreased, suggesting that oxygen radical scavenging in the MT group was associated with MT, although it does not entirely depend on the effects of SOD. Therefore, protecting SOD activity and reducing the content of oxygen free radicals may be one of the mechanisms associated with MT and its protective role in IRI.

In the present study, the mitochondrial respiration rate significantly increased following IR, and a large number of free radicals were produced. Free radicals produced in vivo can easily damage unsaturated fatty acid of lipids in cells, leading to lipid peroxidation. In addition to stabilizing the cell membrane and various other of membranous organelles, MT can protect the mitochondria (30). Mitochondria maintain the physiological function of cells; however they are also the most sensitive organelles to reperfusion injury. This damage presents as transmembrane potential dissipation and disorder of cell energy and metabolism; eventually leading to irreversible cell death. Since mitochondria have a key role in the occurrence and development of IRI, reducing or preventing mitochondrial damage may protect reperfused liver cells. Mitochondrial damage is mainly caused by oxygen free radicals and calcium overload (31) in the process of IR, which mainly presents as a decrease in GSH, which is a mitochondrial antioxidant.

Mitochondrial damage may induce obstacles to oxidative phosphorylation, thus damaging the cells' ability to synthesize ATP. Following reperfusion, the synthesis of ATP remains low, which is not conducive to the recovery of cell function. The present study demonstrated that administration of MT decreased the MDA levels, which may indicate damage to the mitochondrial membrane. This suggests that MT may reduce damage caused by oxygen free radicals and reduce calcium overload. Furthermore, the present study demonstrated that MT increased mitochondrial GSH levels, thus increasing its ability to resist damage. These results suggested that MT may: Maintain the structural integrity of mitochondria, maintain normal cell oxidative phosphorylation function, promote the formation of ATP in reperfused liver cells and reduce the IRI-induced damage in liver cells.

In conclusion, MT may clear oxygen free radicals in three ways: i) Directly combine with free radicals to prevent the chain reaction of free radical oxidation; ii) maintain and protect activity of key enzymes in the antioxidant system, such as glutathione peroxidase and glucose-6-phosphate dehydrogenase, and limit the production of oxygen free radicals (32); and iii) reduce electron leakage and free radical generation in the mitochondrial respiratory chain (33). In particular, the latter two indirect antioxidant effects amplify the protection against damage caused by free radicals.

Serum ALT is highest in the liver. ALT is one of the most sensitive liver function indices (34), as long as $1 \%$ of liver cells undergo necrosis, the serum ALT can be increased by 1 over time. In the present study, as compared with the $\mathrm{N}$ group, ALT levels were significantly increased in the MT and IR groups following IRI, and this increase continued for $24 \mathrm{~h}$ post-reperfusion. As compared with the N group, the levels of AST and $\mathrm{LDH}$, which can reflect acute liver damage, were also raised, demonstrating the destructive effect of IRI on liver function. 
Notably, these three indices for liver function were significantly reduced in the MT group at all time points following reperfusion in the present study, as compared with the IR group. These results suggested that rat liver IRI was significantly reduced following MT treatment. Therefore, exogenous MT may improve liver function following IRI, and may have a protective role in reperfusion injury caused by hepatic portal occlusion.

In conclusion, the present study demonstrated that MT treatment may effectively remove oxygen free radicals, thus reducing liver function damage; attenuate the increased levels of MDA, and decrease the levels of SOD and GSH caused by hepatic IR. Therefore the administration of MT may create favorable conditions for the recovery of liver function following IRI, confirming MT has strong antioxidant properties. Furthermore, no serious side effects of MT have been demonstrated yet; therefore, MT may be a promising therapeutic agent that offers clinical protection from reperfusion injury in the liver.

\section{Acknowledgements}

This study was supported by a grant from the Scientific Research Innovation Project of Shanghai Education Committee, China (no. 13yz040) and by the Shanghai Municipal Commission of Health and Family Planning Fund (award no. 20154Y0207).

\section{References}

1. Ebrahimkhani MR, Daneshmand A, Mazumder A, Allocca M, Calvo JA, Abolhassani N, Jhun I, Muthupalani S, Ayata C and Samson LD: Aag-initiated base excision repair promotes ischemia reperfusion injury in liver, brain, and kidney. Proc Natl Acad Sci USA 111: E4878-E4886, 2014.

2. Rao J, Qin J, Qian X, Lu L, Wang P, Wu Z, Zhai Y, Zhang F, Li G and Wang X: Lipopolysaccharide preconditioning protects hepatocytes from ischemia/reperfusion injury (IRI) through inhibiting ATF4-CHOP pathway in mice. PLoS One 8: e65568, 2013.

3. García JJ,López-Pingarrón L, Almeida-Souza P, Tres A, Escudero P, García-Gil FA, Tan DX, Reiter RJ, Ramírez JM and Bernal-Pérez M: Protective effects of melatonin in reducing oxidative stress and in preserving the fluidity of biological membranes: A review. J Pineal Res 56: 225-237, 2014.

4. Acuña-Castroviejo D, Escames G, Venegas C, Díaz-Casado ME, Lima-Cabello E, López LC, Rosales-Corral S, Tan DX and Reiter RJ: Extrapineal melatonin: Sources, regulation, and potential functions. Cell Mol Life Sci 71: 2997-3025, 2014.

5. Martins PN and Markmann JF: Age-related differences in hepatic ischemia/reperfusion: Gene activation, liver injury, and protective effect of melatonin. J Surg Res 85: e19-e21, 2013.

6. Frich L, Mala T and Gladhaug IP: Hepatic radiofrequency ablation using perfusion electrodes in a pig model: Effect of the Pringle manoeuvre. Eur J Surg Oncol 32: 527-532, 2006.

7. Koh PO: Melatonin prevents hepatic injury-induced decrease in Akt downstream targets phosphorylations. J Pineal Res 51: 214-219, 2011.

8. Aydogan MS, Erdogan MA, Polat A, Yücel A, Ozgül U, Parlakpinar H, Duran ZR, Yildiz A and Durmus M: Protective effects of melatonin and $\beta$-d-glucan against liver injury in rats - a comparative study. Adv Clin Exp Med 22: 621-627, 2012.

9. Kireev R, Bitoun S, Cuesta S, Tejerina A, Ibarrola C, Moreno E, Vara E and Tresguerres JA: Melatonin treatment protects liver of Zucker rats after ischemia/reperfusion by diminishing oxidative stress and apoptosis. Eur J Pharmacol 701: 185-193, 2013.

10. Kireev RA, Cuesta S, Ibarrola C, Bela T, Gonzalez EM, Vara E and Tresguerres JA: Age-related differences in hepatic ischemia/reperfusion: Gene activation, liver injury, and protective effect of melatonin. J Surg Res 178: 922-934, 2012.

11. Kang JW and Lee SM: Melatonin inhibits type 1 interferon signaling of toll-like receptor 4 via heme oxygenase-1 induction in hepatic ischemia/reperfusion. J Pineal Res 53: 67-76, 2012.

12. Kang JW, Koh EJ and Lee SM: Melatonin protects liver against ischemia and reperfusion injury through inhibition of toll-like receptor signaling pathway. J Pineal Res 50: 403-411, 2011.
13. Selvam NT, Venkatakrishnan V, Dhamodharan R, Murugesan S and Kumar SD: Hepatoprotective activity of methanolic extract of Syzygium jambos (Linn.) leaf against paracetamol intoxicated Wistar albino rats. Ayu 34: 305-308, 2013.

14. Jin LM, Jin SF, Liu YX, Zhou L, Xie HY, Yan S, Xu X and Zheng SS: Ischemic preconditioning enhances hepatocyte proliferation in the early phase after ischemia under hemi-hepatectomy in rats. Hepatobiliary Pancreat Dis Int 11: 521-526, 2012.

15. Pires KM, Lanzetti M, Rueff-Barroso CR, Castro P, Abrahão A, Koatz VL, Valença SS and Porto LC: Oxidative damage in alveolar macrophages exposed to cigarette smoke extract and participation of nitric oxide in redox balance. Toxicol In Vitro 26: 791-798, 2012.

16. Papadopoulos D, Siempis T, Theodorakou E and Tsoulfas G: Hepatic ischemia and reperfusion injury and trauma: Current concepts. Arch Trauma Res 2: 63-70, 2013.

17. Peralta C, Jiménez-Castro MB and Gracia-Sancho J: Hepatic ischemia and reperfusion injury: Effects on the liver sinusoidal milieu. J Hepatol 59: 1094-1106, 2013

18. Jaeschke $\mathrm{H}$ and Woolbright BL: Current strategies to minimize hepatic ischemia-reperfusion injury by targeting reactive oxygen species. Transplant Rev (Orlando) 26: 103-114, 2012.

19. Jaeschke H: Molecular mechanisms of hepatic ischemia-reperfusion injury and preconditioning. Am J Physiol Gastrointest Liver Physiol 284: G15-G26, 2003.

20. Mendes-Braz M, Elias-Miró M, Jiménez-Castro MB, Casillas-Ramírez A, Ramalho FS and Peralta C: The current state of knowledge of hepatic ischemia-reperfusion injury based on its study in experimental models. J Biomed Biotechnol 2012: 298657, 2012.

21. Bai J, Dong L, Song Z, Ge H, Cai X, Wang G and Liu P: The role of melatonin as an antioxidant in human lens epithelial cells. Free Radic Res 47: 635-642, 2013.

22. Vishwas DK, Mukherjee A, Haldar C, Dash D and Nayak MK: Improvement of oxidative stress and immunity by melatonin: An age dependent study in golden hamster. Exp Gerontol 48: 168-182, 2013.

23. Bandyopadhyay D, Biswas K, Bandyopadhyay U, Reiter RJ and Banerjee RK: Melatonin protects against stress-induced gastric lesions by scavenging the hydroxyl radical. J Pineal Res 29: $143-151,2000$.

24. Dominguez-Rodriguez A and Abreu-Gonzalez P: Myocardial ischemia-reperfusion injury: Possible role of melatonin. World J Cardiol 2: 233-236, 2010.

25. Elias-Miró M, Jiménez-Castro MB, Rodés J and Peralta C: Current knowledge on oxidative stress in hepatic ischemia/reperfusion. Free Radic Res 47: 555-568, 2013.

26. van Golen RF, van Gulik TM and Heger M. Mechanistic overview of reactive species-induced degradation of the endothelial glycocalyx during hepatic ischemia/reperfusion injury. Free Radic Biol Med 52: 1382-1402, 2012.

27. Palladini G, Ferrigno A, Rizzo V, TarantolaE, Bertone V, Freitas I, Perlini S, Richelmi P and Vairetti M: Lung matrix metalloproteinase activation following partial hepatic ischemia/reperfusion injury in rats. ScientificWorld Journal 2014: 867548, 2014.

28. Parlaktas BS, Atilgan D, Ozyurt H, Gencten Y,Akbas A, Erdemir F and Uluocak N: The biochemical effects of ischemia-reperfusion injury in the ipsilateral and contralateral testes of rats and the protective role of melatonin. Asian J Androl 16: 314-318, 2014.

29. Kerem H, Akdemir O, Ates U, Uyanıkgıl Y, Demırel Sezer E, Bilkay U, Turgut M, Sozmen E and Songur E: The effect of melatonin on a dorsal skin flap model. J Invest Surg 27: 57-64 2014.

30. Ortiz F, García J A, Acuña-Castroviejo D, Doerrier C, López A, Venegas C, Volt H, Luna-Sánchez M, López LC and Escames G: The beneficial effects of melatonin against heart mitochondrial impairment during sepsis: Inhibition of iNOS and preservation of nNOS. J Pineal Res 56: 71-81, 2014

31. Hernández-Esquivel L, Pavón N, Buelna-Chontal M, González-Pacheco H, Belmont J and Chávez E: Citicoline (CDP-choline) protects myocardium from ischemia/ reperfusion injury via inhibiting mitochondrial permeability transition. Life Sci 96: 53-58, 2014.

32. Wang P, Yin L, Liang D, Li C, Ma F and Yue Z: Delayed senescence of apple leaves by exogenous melatonin treatment: Toward regulating the ascorbate-glutathione cycle. J Pineal Res 53: 11-20, 2012.

33. Reiter RJ, Tan DX, Manchester LC, and El-Sawi MR: Melatonin reduces oxidant damage and promotes mitochondrial respiration: Implications for aging. Ann NY Acad Sci 959: 238-250, 2002.

34. Lhuillier F, Parmantier P, Goudable J, Crova P, Delafosse B, Annat G, Cespuglio R and Viale JP: Hepatic ischemia is associated with an increase in liver parenchyma nitric oxide that is in part enzyme-independent. Anesthesiology 98: 373-378, 2003. 